\title{
Two-stage sequential sampling for two rare species in western Inner Mongolia of China
}

\author{
SHI JingJing ${ }^{1,2}$, ZHAO TianZhong ${ }^{1 *} \&$ LEI YuanCai ${ }^{2 *}$ \\ ${ }^{1}$ School of Information Science and Technology, Beijing Forestry University, Beijing 100083, China; \\ ${ }^{2}$ Research Institute of Forest Resource Information Techniques, Chinese Academy of Forestry, Beijing 100091, China
}

Received March 28, 2012; accepted May 10, 2012; published online June 22, 2012

\begin{abstract}
To evaluate the efficiencies of different sampling methods for a rare and clustered population, we investigated the sampling effects for the two species Tamarix chinensis (Salt cedar) and Elaeagnus angustifolia (Russian olive) in western Inner Mongolia with two-stage sequential sampling, which is a new sampling method, traditional simple random sampling and two-stage sampling. Based on two-stage sequential sampling and two-stage sampling, each population was partitioned into four primary sampling units, and then two of them were randomly selected. Sampling designs were simulated based on the conditions of five secondary sampling unit areas, two criterion values, five initial secondary sampling units and two sequential secondary sampling units in 1000 repetitions. To evaluate the performance of the sampling designs for each method, the variance and relative error of the density estimates were used. The relative sampling efficiencies of the three sampling methods were compared using the same final sampling sizes. We analyzed the sampling efficiency generated by two-stage sequential sampling and found that it yielded smaller variances than those of simple random sampling and two-stage sampling in all sampling designs, and that two-stage sampling was more efficient than simple random sampling. Density estimates from the two-stage sequential sampling were very close to the true values. We also determined the optimum secondary sampling unit areas for the two species in the two-stage sequential sampling. It was best for Tamarix chinensis and Elaeagnus angustifolia when the secondary sampling unit areas were 200 and $100 \mathrm{~m}^{2}$, respectively.
\end{abstract}

two-stage sequential sampling, two-stage sampling, simple random sampling, rare and clustered population, Tamarix chinensis, Elaeagnus angustifolia

Citation: Shi J J, Zhao T Z, Lei Y C. Two-stage sequential sampling for two rare species in western Inner Mongolia of China. Chin Sci Bull, 2013, 58: 507-516, doi: $10.1007 / \mathrm{s} 11434-012-5308-7$

The number of individuals in a population is a fundamental piece of information for the conservation and management of rare species. However, problems such as biases and limitations often occur when surveying rare and clustered species and estimating their population size because of their rarity and patchy distribution. Traditional sampling methods may lead to the inclusion of a vast majority of quadrats without individual observations, and thus large variances of population estimates. Fortunately, adaptive sampling is a proven and highly efficient way to sample clustered rare events [1-6]. However, the edge units produced by sam-

*Corresponding authors (email: ztz@bjfu.edu.cn; yclei@caf.ac.cn) pling neighborhoods, which contribute little or nothing to the precision of estimates, have proven to limit the efficiency and applicability of adaptive cluster sampling. In addition, navigating among all units in a neighborhood can be logistically difficult [7]. Thus, a new sampling designtwo-stage sequential sampling (TSSS), with efficient estimators for adaptive TSSS, was proposed by Salehi and Smith [8]. TSSS does not require a neighborhood and does not generate edge units in the sampling, but does exploit clustering in the population to find rare events. Various methods for evaluating the proposed sampling design have been tested [9]. Salehi and Smith [8] simulated the sampling of two real biological populations and one artificial popula- 
tion, and concluded that TSSS is easy to implement and can be applied to a wide range of populations, and furthermore, that TSSS is more efficient than conventional sampling in the designed cases. Brown et al. [10] discussed how best to design adaptive two-stage sequential sampling. However, all of the designed cases only considered the effect on sampling efficiency produced by sampling size (the number of sampling units) in a fixed sampling unit area. In fact, the population can be divided into quantities of sampling units with different sampling unit areas when simulation designs are conducted. For TSSS, different initial sampling sizes, different unit areas and different criterion values will all affect the estimation results for a given population. For instance, the estimation efficiency may be good in a given unit area, but not good or better in another unit area. So it is for criterion values. Therefore, it is necessary to further study and analyze the population estimation effects in as many sampling designs (i.e. different sampling sizes, unit areas and criterion values) as possible to investigate the properties of TSSS.

To evaluate the efficiencies of TSSS in different initial sampling sizes, unit areas and criterion values, this study simulated the sampling of two rare species Tamarix chinensis and Elaeagnus angustifolia in western Inner Mongolia of China and compared TSSS with traditional simple random sampling (SRS) and two-stage sampling (TSS). Finally we determined the optimum secondary sampling unit areas for the two species by TSSS.

\section{Data and methods}

The study area is located in western Inner Mongolia along the Huanghe River Valley, which has an area of $1 \mathrm{~km}^{2}$ centered on $40^{\circ} 15^{\prime} 37.8^{\prime \prime} \mathrm{N}$ and $106^{\circ} 56^{\prime} 28.0^{\prime \prime} \mathrm{E}$. The region is typical of areas with rare and clustered desert vegetation such as Elaeagnus angustifolia and Tamarix chinensis. The study area was divided into 100 plots, each of which was $100 \mathrm{~m} \times 100 \mathrm{~m}$. For boundary measurements, the starting points were always the southwest corners of the plots. The

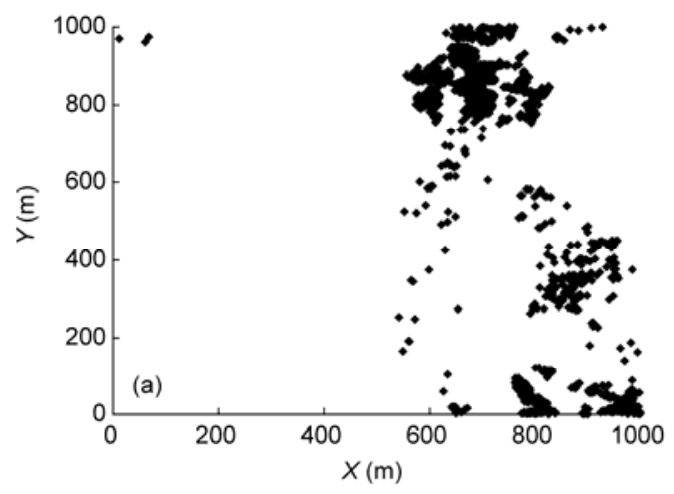

distance between points was measured by an electronic total station, and plot coordinates were recorded by a differential global position system. Each plot was further divided into 100 survey units, each of which was $10 \mathrm{~m} \times 10 \mathrm{~m}$. The border measurement accuracy was $1 / 100$ in principle, namely every $10 \mathrm{~m}$ error for $10 \mathrm{~cm}$. We measured and recorded the ground diameter and height of all desert vegetation (shrubs and trees) in all plots. The spatial distributions of the two investigated species in the survey region are displayed in Figure 1, and it can be seen that their distributions are spatially rare and clustered.

\section{Two-stage sequential sampling}

TSSS is adaptive in the sense that the final sample depends on observed values, but it avoids the use of neighborhoods and the sampling of edge units. The TSSS sampling procedure is as follows.

The population is sampled in two stages. Suppose that a total population has $N_{\mathrm{T}}$ units. The population is partitioned into $M$ primary sampling units (PSUs) and the quantity of secondary sampling units (SSUs) is $N_{i}(i=1,2, \ldots, M)$. Usually the number of $N_{i}$ is the same. Firstly, we choose a sample of $\mathrm{m}$ PSUs from the $M$ PSUs without replacement. Then, we take an initial simple random sample of $n_{i 1}$ units without replacement from PSU $i(i=1,2, \ldots, m)$. Let criterion value $(C)$ be the condition that if satisfied for at least one unit in PSU $i$ causes a predetermined number of additional units $n_{i 2}$ to be selected at random from PSU $i$.

Here Murthy's estimator is used to devise unbiased estimators for the design [11].

The population total is estimated as follows:

$$
\hat{\tau}=\sum_{i=1}^{m} \frac{\hat{\tau}_{i}}{\pi_{i}},
$$

where $\hat{\tau}_{i}$ is the estimator for the sum of the values of the $i$ th PSU, and $\pi_{i}$ is the inclusion probability for PSU $i . m$ is the number of PSUs sampled.

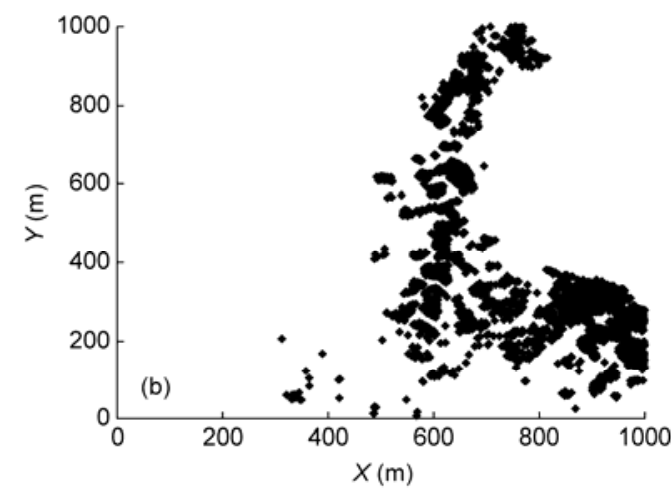

Figure 1 Distributions of the two species. (a) Tamarix chinensis; (b) Elaeagnus angustifolia. 
The variance of $\hat{\tau}$ is

$$
\operatorname{var}[\hat{\tau}]=\sum_{i=1}^{M} \sum_{i^{\prime}=1}^{M}\left(\frac{\pi_{i i^{\prime}}-\pi_{i} \pi_{i^{\prime}}}{\pi_{i} \pi_{i^{\prime}}}\right) \tau_{i} \tau_{i^{\prime}}+\sum_{i=1}^{M} \frac{\operatorname{var}\left[\hat{\tau}_{i}\right]}{\pi_{i}},
$$

where $\pi_{i^{\prime}}$ is the inclusion probability for PSU $i^{\prime} . \pi_{i i^{\prime}}$ is the joint inclusion probability for PSUs $i$ and $i^{\prime} . M$ is the total number of PSUs. $\tau_{i}$ is the sum of the values (e.g. the number of plants) of the $i$ th PSU, $\operatorname{var}\left[\hat{\tau}_{i}\right]$ is the variance of $\hat{\tau}_{i}$.

The unbiased estimator of $\operatorname{var}[\hat{\tau}]$ is

$$
\hat{\operatorname{var}}[\hat{\tau}]=\sum_{i=1}^{m} \sum_{i^{\prime}=1}^{m}\left(\frac{\pi_{i i^{\prime}}-\pi_{i} \pi_{i^{\prime}}}{\pi_{i} \pi_{i^{\prime}}}\right) \frac{\hat{\tau}_{i} \hat{\tau}_{i^{\prime}}}{\pi_{i i^{\prime}}}+\sum_{i=1}^{m} \frac{\operatorname{vâr}\left[\hat{\tau}_{i}\right]}{\pi_{i}},
$$

$\operatorname{vâr}\left[\hat{\tau}_{i}\right]$ is the estimator of variance for $\hat{\tau}_{i}$. The relevant computational formulas for $\hat{\tau}_{i}$ and $\operatorname{vâ}\left[\hat{\tau}_{i}\right]$ are given by Salehi and Smith [8].

\section{Simulation sampling designs}

It is often infeasible to analytically derive the sampling distribution for estimators across a range of populations and designs. The results reckoned by different samples are different and also differ from the true value. Thus, the result obtained by a once-only sample cannot confirm whether the sampling method is good. We should compare various sampling methods in as many repetitions as possible.

To permit experimental comparison across populations and designs, a simulation study was conducted to evaluate the sampling designs. Simulation makes it possible to evaluate the sampling distribution of the estimators based on repeated sampling. Comparisons across multiple populations and a broad range of designs can result in robust recommendations [12,13].

Considering the characteristics of rare, aggregate populations, and the results of the relative error of density estimates in many repetitions, the simulation study designed the least number of sampling units as 50 and the smallest SSU area as $5 \mathrm{~m} \times 5 \mathrm{~m}$.

The SSUs mainly did not expand when $C$ increased to 3 or 4. As $C$ continued to increase, the initial population was close to that of SRS. So the largest $C$ was no more than 2 .
Thus, $C$ was set at 1 or 2 . When the value of a selected unit was equal to or greater than $C$, a quantity of additional units is added to the sample.

In the simulation designs for the total population, we considered that the relative density errors estimated by TSSS were less than 5\% in different SSU area designs, and that the mean density estimates were invariable as repetitions increased to 1000 . The different sampling designs are presented in Table 1.

In the simulation study, sampling without replacement was replicated 1000 times and the sampling units were square. The two populations (Tamarix chinensis and Elaeagnus angustifolia) were partitioned into four PSUs, and then two of the PSUs were randomly selected. TSSS was simulated in $5 \mathrm{SSU}$ area designs $\left(25 \mathrm{~m}^{2}(5 \mathrm{~m} \times 5 \mathrm{~m}), 100 \mathrm{~m}^{2}\right.$ $(10 \mathrm{~m} \times 10 \mathrm{~m}), 200 \mathrm{~m}^{2}(10 \mathrm{~m} \times 20 \mathrm{~m}), 400 \mathrm{~m}^{2}(20 \mathrm{~m} \times 20 \mathrm{~m})$, $\left.500 \mathrm{~m}^{2}(20 \mathrm{~m} \times 25 \mathrm{~m})\right), 5$ initial SSUs (SSU_ $n_{1}=50,100$, 150, 200 and 250) and 2 sequential SSUs (SSU_ $n_{2}=40$ and 80). For traditional TSS, without sequential SSUs, the sampling stages were very similar to those of TSSS, while SRS proceeded in only one stratum. The final sampling sizes of TSS and SRS were the same as those (the units sampled $N=$ SSU_ $n_{1}+$ SSU_ $n_{2}$ ) of TSSS.

\section{Evaluated indicators}

The survey data for the simulation study were imported into the software SAMPLE (it can be downloaded from http: //www.lsc.usgs.gov/aeb/davids/acs/) to simulate sampling. To evaluate the performance of the different sampling designs, the variance $(E(v))$ and relative error of the density estimate were used. We evaluated the different sampling methods by their relative sampling efficiency. Relative sampling efficiency is the ratio of variance from a traditional sampling design to variance from a candidate design with equal final sampling sizes. The relevant formulas are as follows:

The density estimator and the variance for TSSS in the $i$ th sampling occasion are respectively $\hat{\mu}_{i}$ and $\operatorname{var}\left(\hat{\mu}_{i}\right)(i$ $=1,2, \ldots, n)$.

$$
\hat{\mu}_{i}=\frac{\hat{\tau}}{A}
$$

\begin{tabular}{|c|c|c|c|c|c|}
\hline Total area $\left(\mathrm{m}^{2}\right)$ & $N$ & $N \_$PSU & S_PSU $\left(\mathrm{m}^{2}\right)$ & $N \_\mathrm{SSU}$ & S_SSU $\left(\mathrm{m}^{2}\right)$ \\
\hline 1000000 & 40000 & 4 & 250000 & 10000 & $25(5 \times 5)$ \\
\hline 1000000 & 10000 & 4 & 250000 & 2500 & $100(10 \times 10)$ \\
\hline 1000000 & 5000 & 4 & 250000 & 1250 & $200(10 \times 20)$ \\
\hline 1000000 & 2500 & 4 & 250000 & 625 & $400(20 \times 20)$ \\
\hline 1000000 & 2000 & 4 & 250000 & 500 & $500(20 \times 25)$ \\
\hline
\end{tabular}

Table 1 Designs for simulation sampling ${ }^{\text {a) }}$

a) $N$, total number of population units; N_PSU, number of PSU; S_PSU, area of PSU; N_SSU, number of SSU; S_SSU, area of SSU. 
$\hat{\tau}$ is the estimator for the population total, which is calculated by eq. (1). A is the total study area.

$$
E(\hat{\mu})=\frac{\sum_{i=1}^{n} \hat{\mu}_{i}}{n} .
$$

$E(\hat{\mu})$ expresses the mean density estimated in certain repetitions. $n$ represents the number of repetitions.

The variance of the density estimates for TSSS in ith simulated sampling occasion is:

$$
\operatorname{var}\left(\hat{\mu}_{i}\right)=\frac{\operatorname{vâr}_{i}(\hat{\tau})}{A^{2}}
$$

$\operatorname{vâr}_{i}(\hat{\tau})$, which is calculated by equation (3), is the variance of $\hat{\tau}$ in the ith sampling occasion. For TSS and SRS, the density in the $i$ occasion sampling is $\hat{\mu}_{i}=\frac{N_{T}}{A} \hat{y}_{\text {TSS }, \mathrm{SRS}}$, where $N_{\mathrm{T}}$ is the total number of sampling units in the population, as defined above. $\hat{y}_{\text {TSS }}$ and $\hat{y}_{\mathrm{SRS}}$ are the values of each sampling unit estimated by TSS and SRS methods, respectively. Their computational formulas and the variance estimators can be found in Song [14].

The variance of density estimates in $\mathrm{n}$ repetitions is:

$$
E(v)=\frac{\sum_{i=1}^{n} \operatorname{var}\left(\hat{\mu}_{i}\right)}{n} .
$$

The relative sampling efficiency is:

$$
\operatorname{Efficiency}(\hat{\mu})=E_{\bar{y}}(v) / E_{\hat{\mu}}(v),
$$

$\hat{\mu}$ is the population density estimated by TSSS. $E_{\bar{y}}(v)$ is the variance of the density estimated by a traditional method with the same final sampling size as TSSS. $E_{\hat{\mu}}(v)$ is the variance of the density estimated by TSSS.

The formula for the relative error of the density estimate is

Relative error of density estimate

$$
=\frac{\mid \text { Density estimate }- \text { True density } \mid}{\text { True density }} \text {. }
$$

\section{Results and discussion}

Through the simulation study, the densities estimated by TSSS were close to the true densities in different sampling designs. The true densities of Tamarix chinensis and Elaeagnus angustifolia were 0.00194 and $0.0034 \mathrm{~m}^{-2}$, respectively. The biggest relative errors of the density estimates were $3.714 \%$ for Tamarix chinensis and $2.567 \%$ for Elaeagnus angustifolia, and the results indicated that the differences between the estimated and true densities were small. The mean relative errors of the density estimates for TSSS were $1.3 \%$ for Tamarix chinensis and $0.689 \%$ for Elaeagnus angustifolia in 5 initial SSU_n $n_{1}, 2$ SSU_ $n_{2}$ and 5 SSU areas when $C$ was 1 to 2 . The relative errors of the density estimates for all simulation designs were smaller than 5\% (Figure 2).

Some related studies did not consider the relative errors and variance estimates for TSSS itself in various sampling designs. In our study, the relative errors were smaller than $5 \%$, showing that TSSS was suitable for the species studied. Variance estimates can not only judge whether a sampling method is bad or good, but can also measure effects in different sampling designs. Variance estimates in different initial sampling sizes, unit areas and $C$ could be useful to find the regularity for various designs and determine the proper design for the species of interest. The density variances for Tamarix chinensis and Elaeagnus angustifolia estimated by TSSS were less than 0.01 in different simulation designs (Figure 3).

Generally, as shown in Figure 3, in a certain SSU area, $C$ and quantity of PSUs, the larger the number of initial SSU_ $n_{1}$ or SSU_ $n_{2}$ sampled, the smaller the variances acquired. The variances for SSU_ $n_{1}=200$ and SSU $\_n_{2}=250$ were similar. Considering the sampling cost, SSU_n $n_{1}=200$ would be appropriate. It would be better still when SSU_ $n_{2}=$ 80 then SSU $n_{2}=40$. The variances would increase with increasing $C$ in a certain quantity of SSU_ $n_{1}$ and SSU_n $n_{2}$. The variances decreased as the SSU areas increased when $C$ was invariant. Taking Elaeagnus angustifolia for example, the TSSS variances for different SSU areas, $C$ and SSU_ $n_{1}$ when PSUs=2 and SSU $n_{2}=80$, are presented in Figure 4.

TSSS was adaptive in a sense. The condition to adapt was based on the observation of a unit meeting or exceeding a predetermined $C$. Whenever the value of the variable of interest (e.g. the number of plants) of a selected unit satisfied a specified condition, say $C$, then additional units were added into the sample according to the sampling method, which is different from traditional sampling methods. For the results above, generally speaking, for a smaller $C$, the more units with target were likely added to the sample. Thus, the variances were less and the estimations were more accurate. As $C$ increased, the variances increased and the superiority of TSSS was less convincing. Thus, the overall survey may be close to the traditional SRS. Therefore, it was better to design $C=1$ to make good use of TSSS advantages. The population was divided into different SSU areas and as the SSU areas increased, units with larger values were more easily satisfied for $C$. Thus, more targets in units participating in the estimation could enhance the accuracy.

Commonly, a candidate sampling method was compared with traditional sampling techniques by the relative sampling efficiency presented by Thompson and Seber [15], the ratio of variances from the traditional sampling method and TSSS (e.g. variance of SRS divided by variance of TSSS). When the ratio was greater than 1 , the efficiency of TSSS 

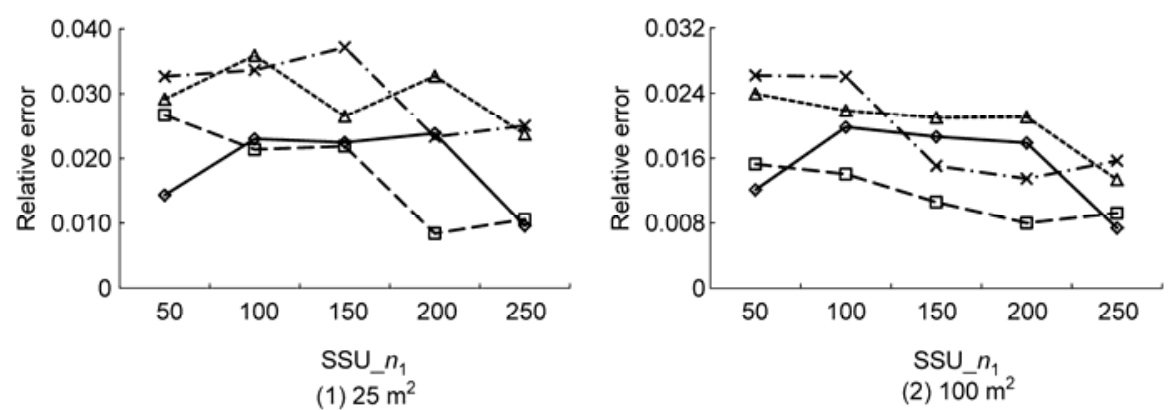

(2) $100 \mathrm{~m}^{2}$

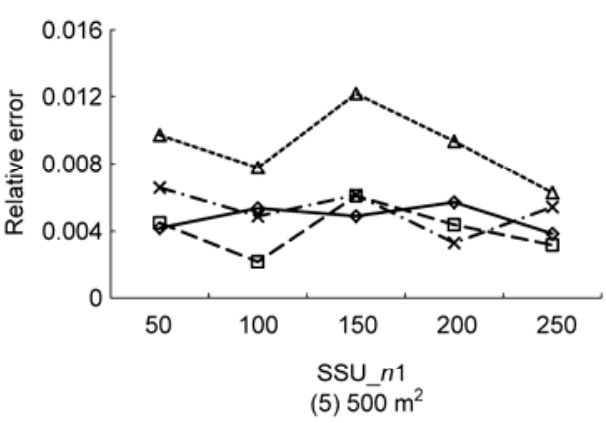

(a) Tamarix chinensis

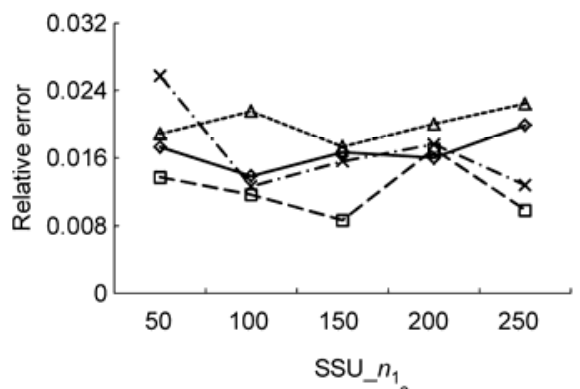

(1) $25 \mathrm{~m}^{2}$

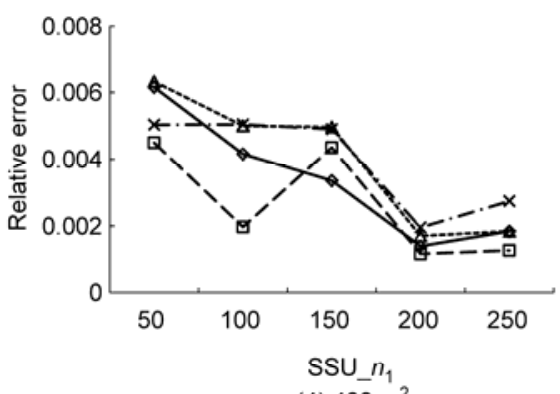

(4) $400 \mathrm{~m}^{2}$

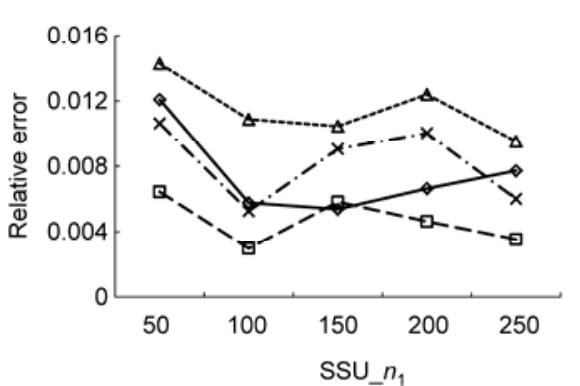

(2) $100 \mathrm{~m}^{2}$

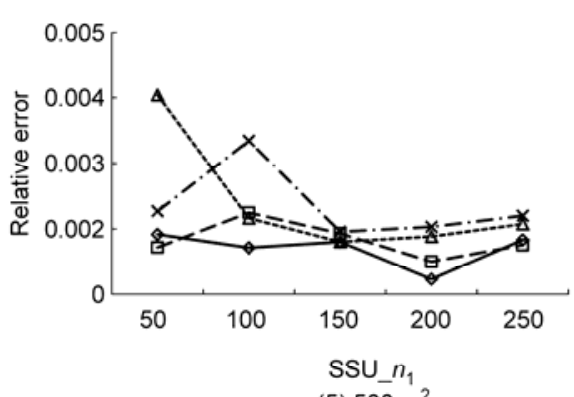

(5) $500 \mathrm{~m}^{2}$

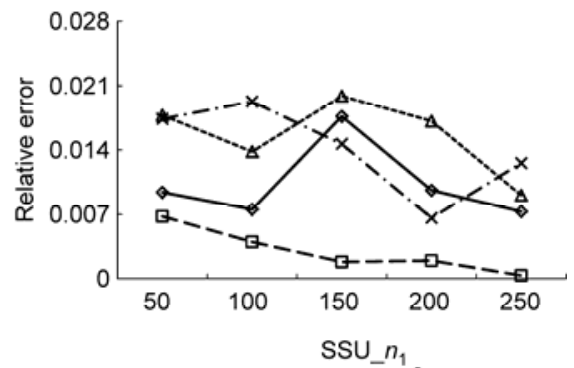

(3) $200 \mathrm{~m}^{2}$

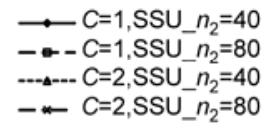

(b) Elaeagnus angustifolia

Figure 2 The relative errors in different SSU areas. $C, \operatorname{SSU} \_n_{1}$ and SSU_n $n_{2}$ for the two species estimated by TSSS.

was higher in the same final sampling size. The efficiencies of TSSS relative to SRS and TSS were usually greater than 1 in different SSU areas, $C$ and quantities of PSUs, SSU_ $n_{1}$ and SSU_ $n_{2}$ (Figures 5 and 6).

In Table 2, Eff ${ }^{a}>1$ and Eff $^{\mathrm{b}}>1$ which indicated that the efficiencies of TSSS were higher than those of SRS and TSS with the same final sampling sizes. The relative errors of the density estimates would be larger when $C$ was enlarged when the other design indices were fixed, while the efficiencies of TSSS relative to SRS and TSS decreased (Table 2; Figures 2, 5 and 6). Relative to TSS with the same sampling sizes, TSSS had higher efficiencies than SRS in different designs.

Very different sampling designs have been examined by 


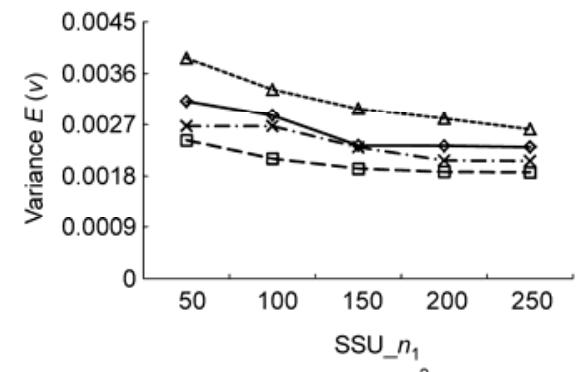

(1) $25 \mathrm{~m}^{2}$

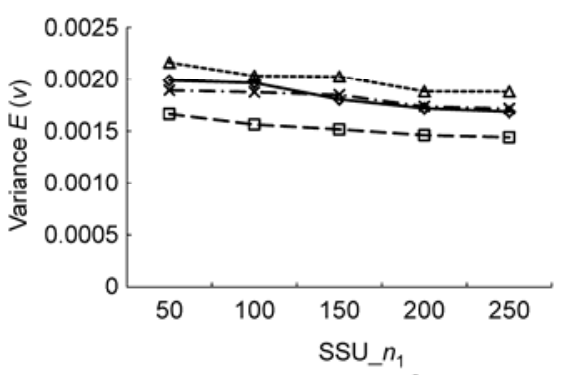

(4) $400 \mathrm{~m}^{2}$

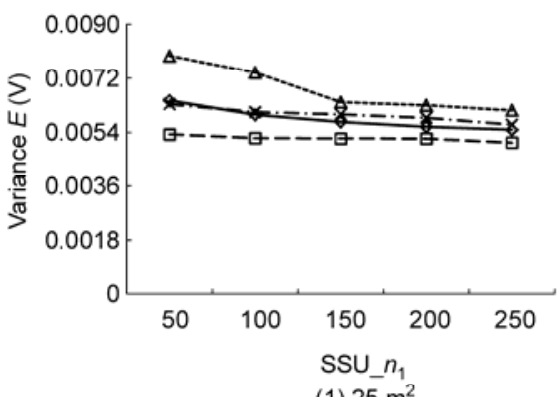

(1) $25 \mathrm{~m}^{2}$

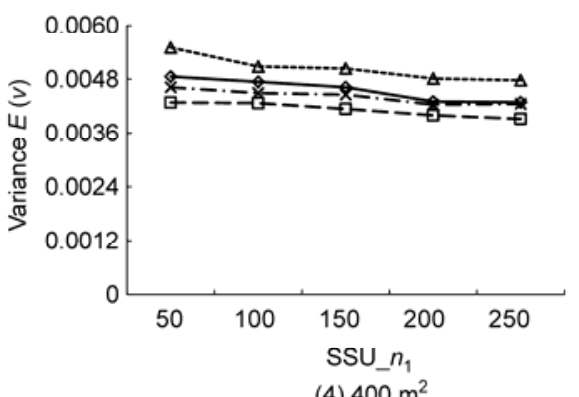

(4) $400 \mathrm{~m}^{2}$

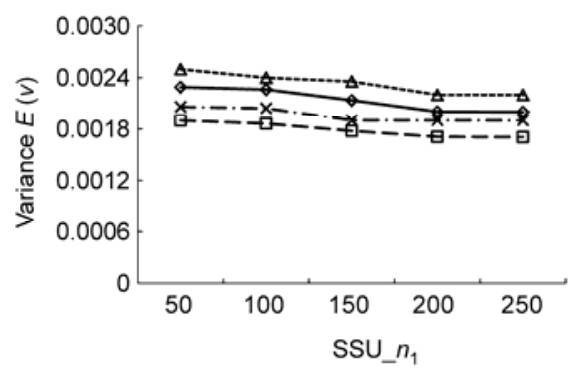

(2) $100 \mathrm{~m}^{2}$

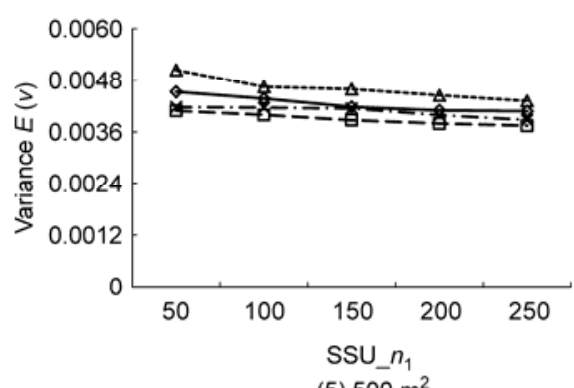

(a) Tamarix chinensis
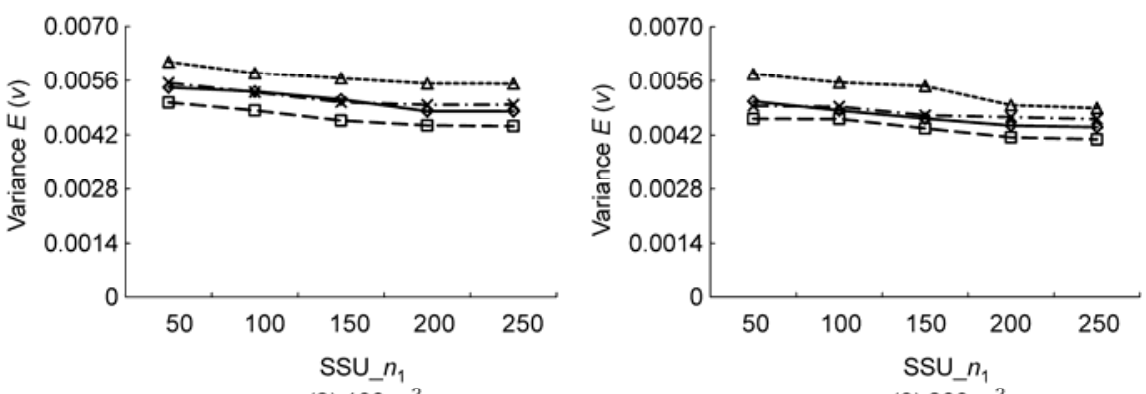

(2) $100 \mathrm{~m}^{2}$

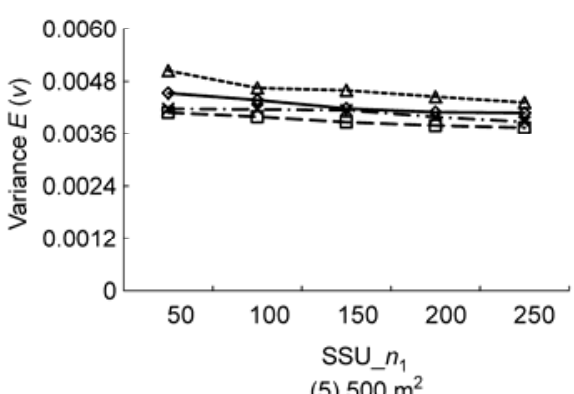

(b) Elaeagnus angustifolia
(3) $200 \mathrm{~m}^{2}$

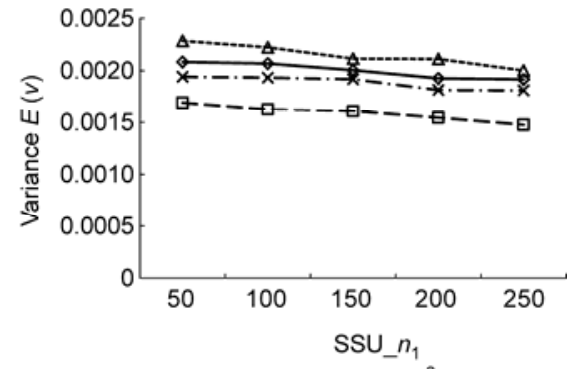

(3) $200 \mathrm{~m}^{2}$

Figure 3 The variances in different SSU areas. $C, \operatorname{SSU} \_n_{1}$ and SSU $n_{2}$ for the two species estimated by TSSS.

different studies. Some simulations studied the sampling effects when the total PSUs were sampled, which is close to stratified sampling [8]. In this study, the quantity of PSUs sampled was fixed and no more than the total number of PSUs. For different populations (species), the sampling efficiency would be different because of their distinctive distributions. Research on the population of freshwater mussels by Salehi and Smith showed that the efficiencies of TSSS relative to traditional sampling methods were no more than 2 [8]. Those for the population of blue-winged teal were no more than 2.5 [10]. In this study, the efficiencies of TSSS increased rapidly as SSU_ $n_{1}$ increased, e.g. the TSSS was never less than 2 times more efficient than SRS for the species studied when SSU_ $n_{1}$ was 100 (Figures 5 and 6). Therefore, 

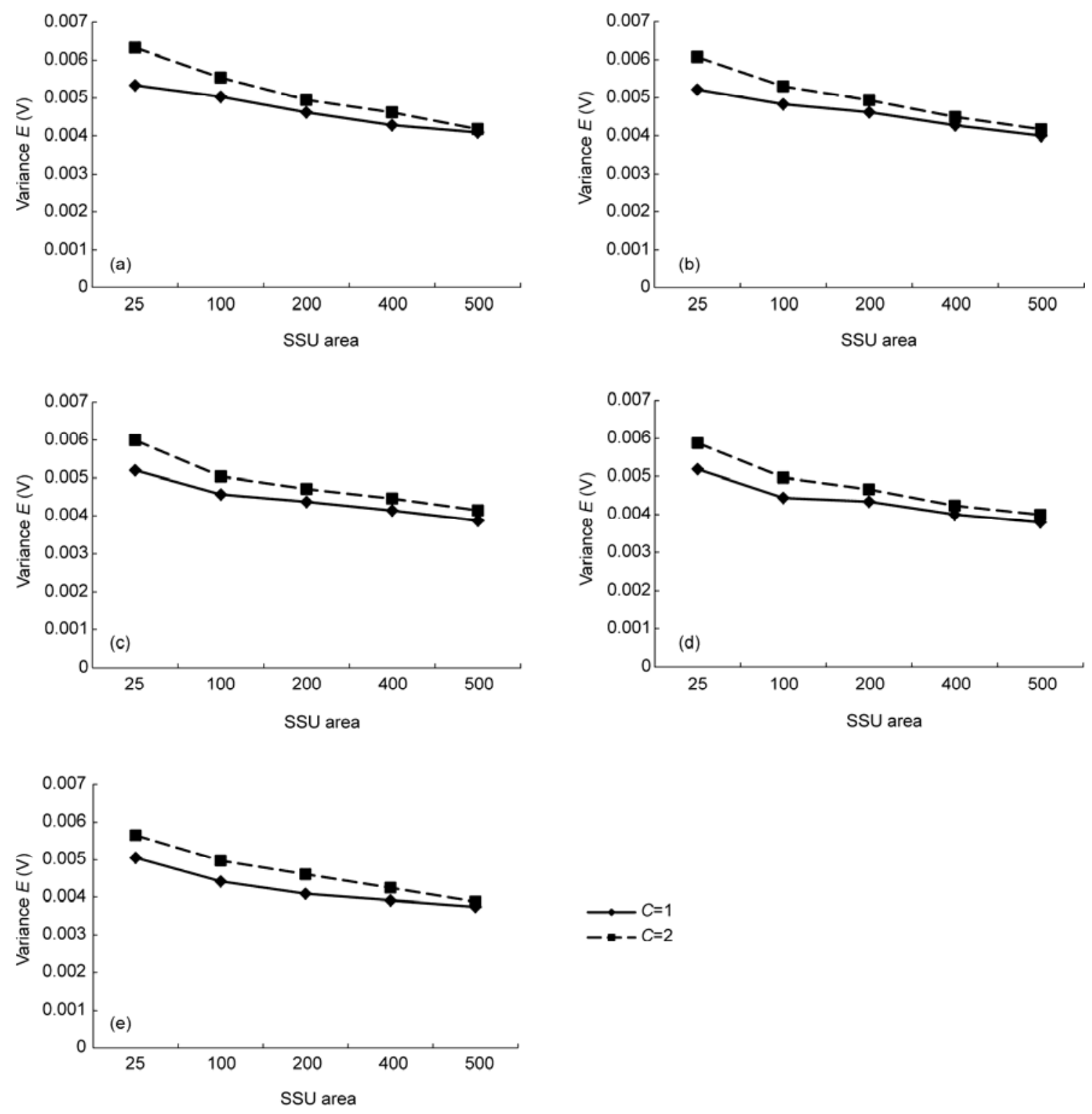

Figure 4 The variances for different SSU areas. $C$ and SSU $\_n_{1}$ when PSUs=2 and SSU $n_{2}=80$ for Elaeagnus angustifolia estimated by TSSS. (a) SSU $\_n_{1}=50$; (b) SSU $\_n_{1}=100$; (c) SSU $n_{1}=150$; (d) SSU $n_{1}=200$; (e) SSU $n_{1}=250$.

for different population distributions, there would be different suitable sampling methods and designs.

The results demonstrated in Figures 1-6 for different SSU areas showed that the sampling effects would be influenced by the sampling unit areas. In some related studies [7-10], the researchers studied the effects of sampling methods in a fixed SSU area. Unlike those studies, the SSU area was not fixed in this study. For the determination of the best unit area in the simulation study, we determined the optimum SSU area for sampling the two targets through analyses of the relative errors of the density estimates for different SSU areas. The designs proceeded with PSUs $=2, \operatorname{SSU} \_n_{2}=80$ and 1000 repetitions when $C$ was 1 in different SSU areas and SSU_ $n_{1}$. The relative errors of TSSS were less than $1 \%$.

Figure 7 shows that as the SSU areas increased, the rela- tive errors of Elaeagnus angustifolia and Tamarix chinensis presented roughly the same change trend. From a visual point of view, the SSU areas less than $100 \mathrm{~m}^{2}$ had greater relative errors. The relative errors decreased as the SSU areas increased.

The simulation sampling of Tamarix chinensis was perfect when the SSU area was $200 \mathrm{~m}^{2}$ (Figure 7(a)). For Elaeagnus angustifolia, the relative errors reduced quickly when the SSU area was $100 \mathrm{~m}^{2}$ (Figure 7(b)). Therefore, the sampling effect was best at $100 \mathrm{~m}^{2}$ for Elaeagnus angustifolia. That meant that the relative errors of the density estimates were larger when the SSU areas were smaller than $100 \mathrm{~m}^{2}$. When the SSU areas were larger than $100 \mathrm{~m}^{2}$, the relative errors decreased, but the magnitudes of reduction also decreased. At the same time, with increasing unit areas, 


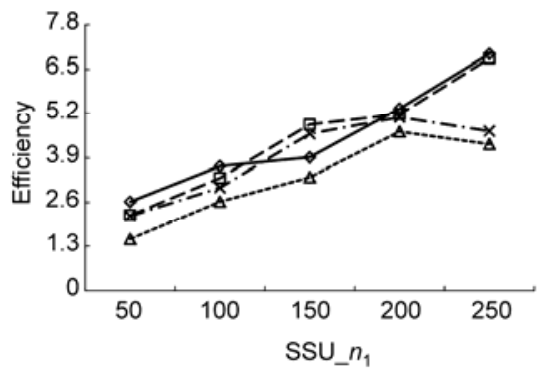

(1) $25 \mathrm{~m}^{2}$

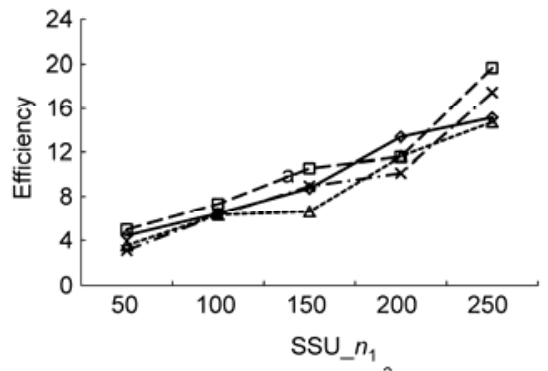

(4) $400 \mathrm{~m}^{2}$
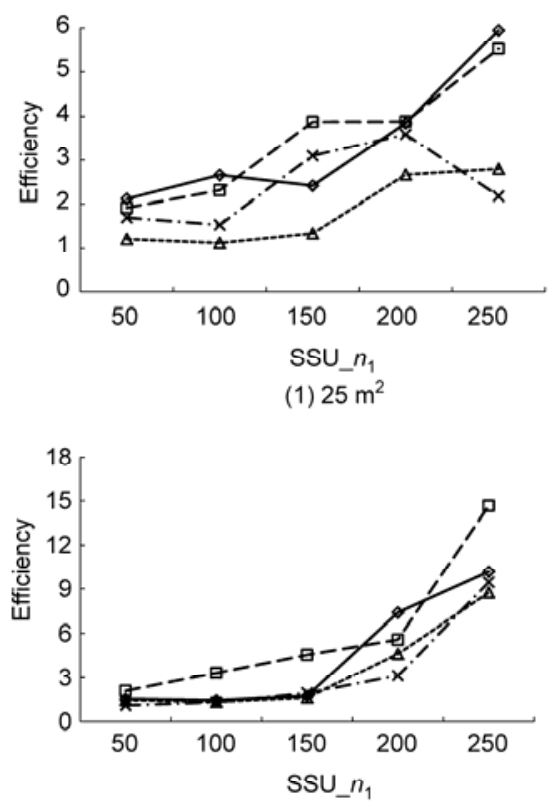

(4) $400 \mathrm{~m}^{2}$

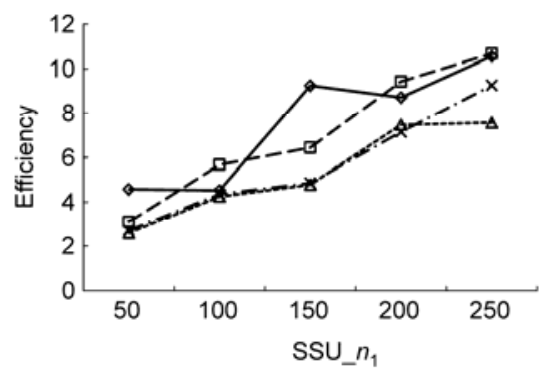

(2) $100 \mathrm{~m}^{2}$

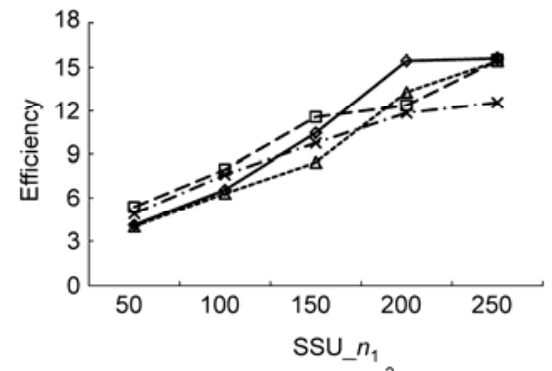

(5) $500 \mathrm{~m}^{2}$

(a) The efficiency for TSSS relative to a SRS

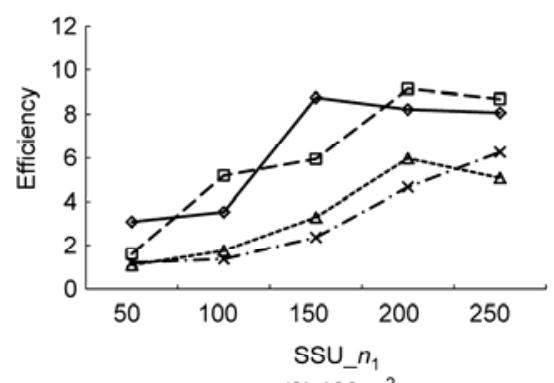

(2) $100 \mathrm{~m}^{2}$

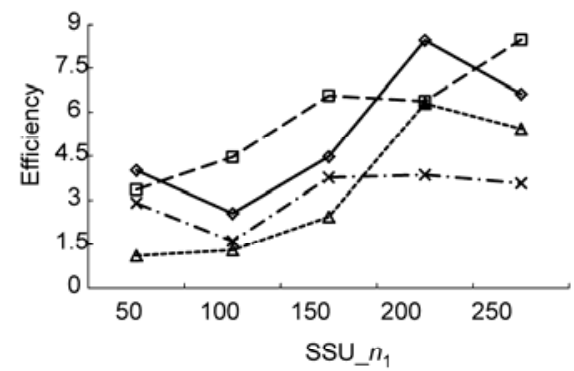

(5) $500 \mathrm{~m}^{2}$

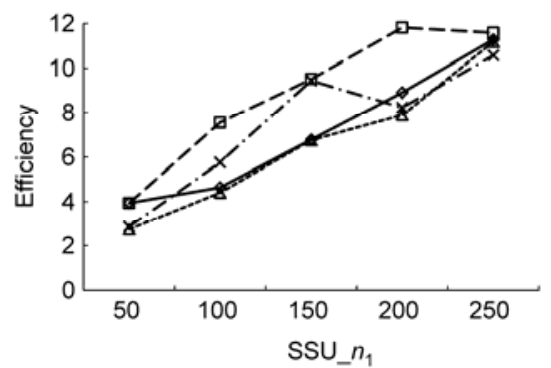

(3) $200 \mathrm{~m}^{2}$

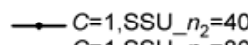

$-C=1, S_{S U}^{-} n_{2}=80$

$\cdots+C=2, S S U+n_{2}=40$

$-\leftarrow C=2, S_{-} n_{2}=80$

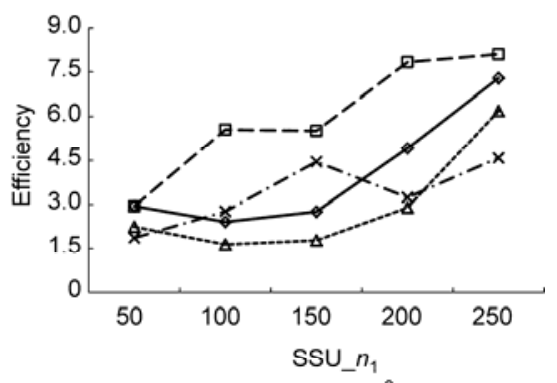

(3) $200 \mathrm{~m}^{2}$

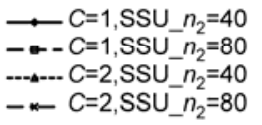

(b) The efficiency for TSSS relative to a TSS

Figure 5 The relative sampling efficiencies of TSSS in different SSU areas. $C, \operatorname{SSU} \_n_{1}$ and SSU $n_{2}$ for Tamarix chinensis.

measurement costs would increase.

\section{Conclusions}

In a certain quantity of PSUs, SSU_n $n_{1}, \mathrm{SSU} \_n_{2}$, SSU area and $C$, the variance of TSSS is smaller than those of TSS and SRS. The TSSS density estimates are very close to the true values. Based on the same final sampling sizes, we know from the above analyses that TSSS is more efficient than the traditional SRS and TSS, and that TSS is more efficient than SRS.

The PSU area affects both the SSU size and the final sampling size for TSSS designs. Only different SSU areas are studied here, but the PSU area is fixed. Thus, the PSU area that impacts sampling efficiency should be further studied. TSSS is one of the adaptive sampling designs used to control the final sampling size. Inverse sampling and 


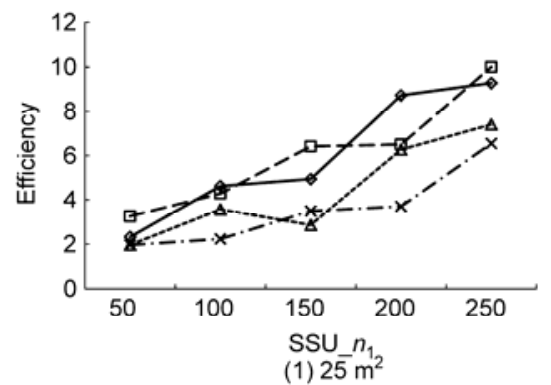

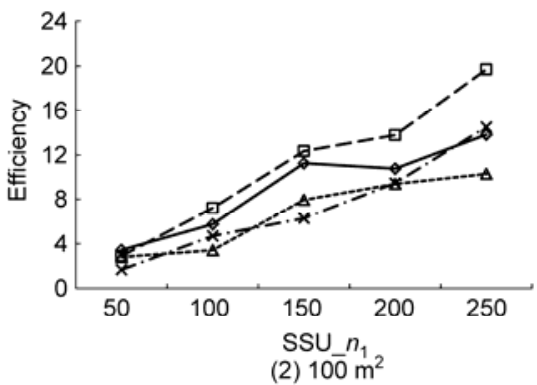

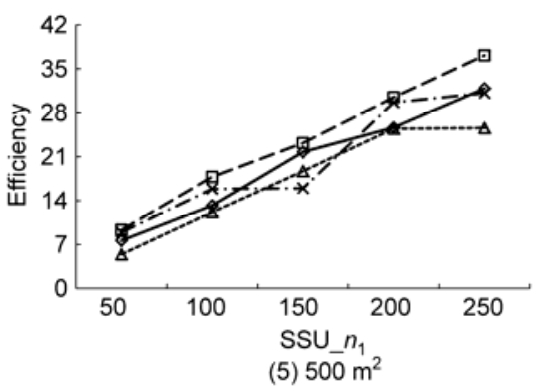

(a) The efficiency for TSSS relative to a SRS

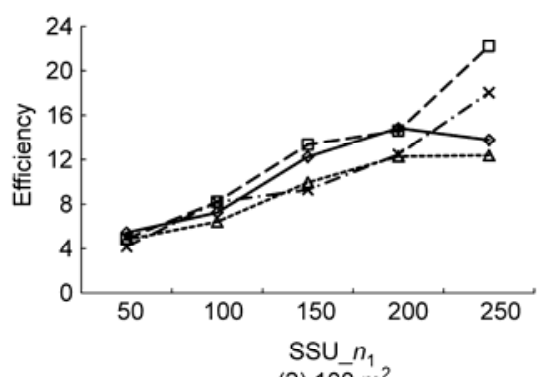

(2) $100 \mathrm{~m}^{2}$

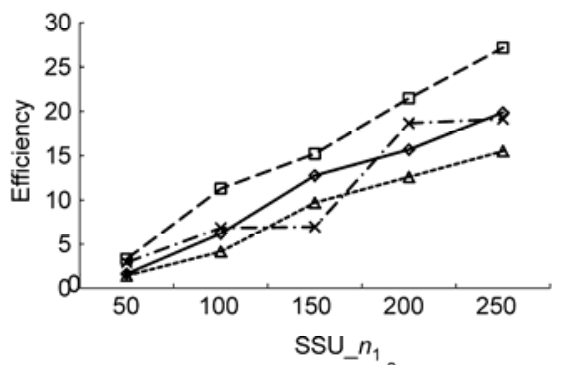

(5) $500 \mathrm{~m}^{2}$

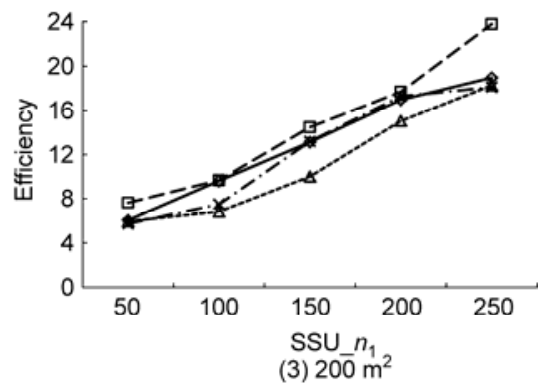

(3) $200 \mathrm{~m}^{2}$

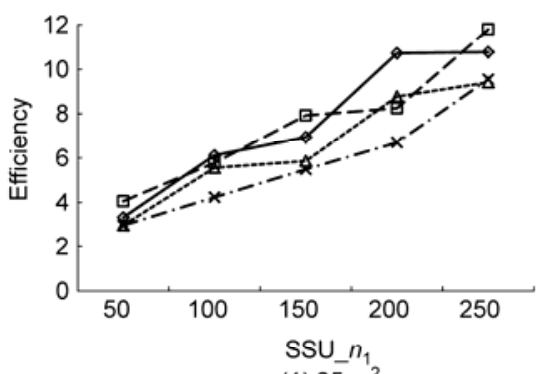

(1) $25 \mathrm{~m}^{2}$

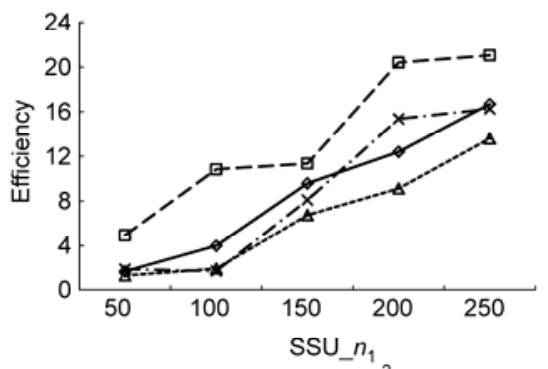

(4) $400 \mathrm{~m}^{2}$

(b) The efficiency for TSSS relative to a TSS

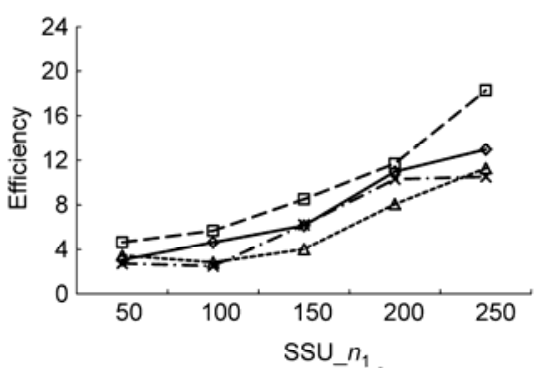

(3) $200 \mathrm{~m}^{2}$

Figure 6 The relative sampling efficiencies of TSSS in different SSU areas. $C, \operatorname{SSU} \_n_{1}$ and SSU_ $n_{2}$ for Elaeagnus angustifolia.

Table 2 Relative sampling efficiencies, density and variance estimates when the initial SSU $\_n_{1}=200, \mathrm{SSU} \_n n_{2}=80$ and SSU area was $25 \mathrm{~m}^{2}$ for the two species ${ }^{\text {a) }}$

\begin{tabular}{|c|c|c|c|c|c|c|c|c|c|}
\hline \multirow{3}{*}{ Species } & \multicolumn{9}{|c|}{ Sampling method } \\
\hline & \multicolumn{3}{|c|}{ SRS } & \multicolumn{3}{|c|}{ TSS } & \multicolumn{3}{|c|}{ TSSS } \\
\hline & $D$ & Eff $^{\mathrm{a}}$ & $E(v)$ & $D$ & $\mathrm{Eff}^{\mathrm{b}}$ & $E(v)$ & $D$ & $E(v)$ & $N_{\mathrm{F}}$ \\
\hline $\mathrm{TC}(C=1)$ & 0.00186 & 5.182 & 0.0097 & 0.00203 & 3.880 & 0.0073 & 0.00193 & 0.00187 & 483 \\
\hline $\mathrm{TC}(C=2)$ & 0.00205 & 5.092 & 0.0105 & 0.00204 & 3.590 & 0.0074 & 0.00199 & 0.00207 & 395 \\
\hline $\operatorname{EA}(C=1)$ & 0.00329 & 8.245 & 0.0427 & 0.00331 & 6.505 & 0.0337 & 0.00346 & 0.00518 & 492 \\
\hline $\operatorname{EA}(C=2)$ & 0.00364 & 6.696 & 0.0393 & 0.00325 & 3.695 & 0.0217 & 0.00334 & 0.00588 & 400 \\
\hline
\end{tabular}

a) TC, Tamarix chinensis; EA, Elaeagnus angustifolia; $\mathrm{Eff}^{\mathrm{a}}, \operatorname{Var}_{(\mathrm{SRS})} / \operatorname{Var}_{(\mathrm{TSSS})} ; \mathrm{Eff}^{\mathrm{b}}, \operatorname{Var}_{(\mathrm{TSS})} / \mathrm{Var}_{(\mathrm{TSSS})} ; \mathrm{D}$, density; $E(v)$, variance; $N_{\mathrm{F}}$, final units sampled over 1000 replications. 


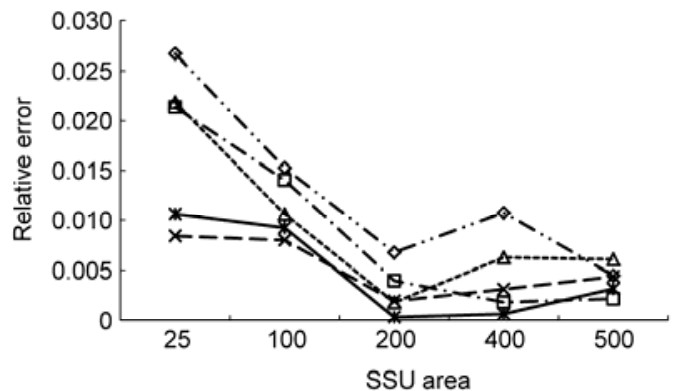

(a) Tamarix chinensis

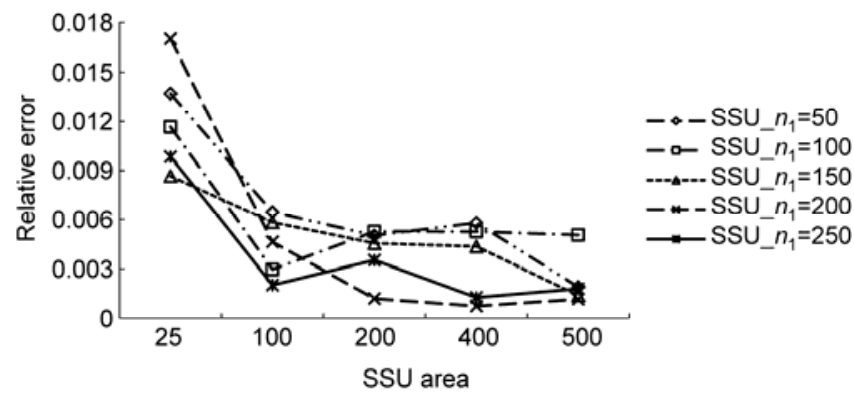

(b) Elaeagnus angustifolia

Figure 7 The relative errors of the density estimates estimated by TSSS for different SSU areas when PSUs=2, SSU $\_n_{2}=80$ and $C=1$.

restricted adaptive cluster sampling have also been presented to address the problem of sampling size confirmation [1,16-18]. An evaluation of the efficiencies between TSSS and those methods is worth carrying out.

How to design an efficient survey is still an interesting question for the study of rare and clustered populations. We should not always launch an investigation by one sampling method alone. Combining one method with another to survey a population may be more efficient. Thus, a combination of TSSS with some new methods or traditional design such as systemic sampling should be considered.

This work was supported by State Forestry Administration (201204510 and 200904003), National Natural Science Foundation of China (31170588) and Social commonweal Research Progvams of Ministry of Science and Technology (2005DIB5J142). The authors thank Dr. David Smith in USGS for providing information and software.

1 Brown J A. The application of adaptive cluster sampling to ecological studies. In: Fletcher D J, Manly B F J, eds. Statistics in Ecology and Environmental Monitoring. Dunedin: University of Otego Press, 1994. 86-97

2 Philippi T. Adaptive cluster sampling for estimation of abundances within local populations of low-abundance plants. Ecology, 2005, 86: 1091-1100

3 Roesch F A Jr. Adaptive cluster sampling for forest inventories. For Sci, 1993, 39: 655-669

4 Smith D R, Conroy M J, Brakhage D H. Efficiency of adaptive cluster sampling for estimating density of Wintering Waterfowl. Biometrics, 1995, 51: 777-788
5 Magnussen S, Kurz W, Leckie D G, et al. Adaptive cluster sampling for estimation of deforestation rates. Eur J For Res, 2005,124: 207-220

6 Shi J J, Lei Y C, Zhao T Z. Comparison between adaptive cluster sampling and simple random sampling: A case study of coastal Bruguiert gymnorrhiza (in Chinese). J Northeast For Univ, 2011, 39: 105-106

7 Salehi M M. Rao-Blackwell versions of the Horvitz-Thompson and Hansen-Hurwitz in adaptive cluster sampling. Environ Ecol, 1999, 6: 183-195

8 Salehi M M, Smith D R. Two-stage sequential sampling: A neighborhood-free adaptive sampling procedure. J Agric Biol Environ Stat, 2005, 10: 84-103

9 Salehi M, Moradi M, Brown J A, et al. Efficient estimators for adaptive stratified sequential sampling. J Stat Comput Simul, 2010, 80: 1163-1179

10 Brown J A, Salehi M M, Moradi M, et al. An adaptive two-stage sequential design for sampling rare and clustered populations. Soc Ecol Popul Springer, 2008, 50: 239-245

11 Murthy M N. Ordered and unordered estimators in sampling without replacement. Sankhya, 1957, 18: 379-390

12 Brown J A. Designing an efficient adaptive cluster sample. Environ Ecol Stat, 2003, 10: 95-105

13 Morrison L W, Smith D R, Young C C, et al. Evaluating sampling designs by computer simulation: A case study with the Missouri bladderpod. Popul Ecol, 2008, 50: 417-425

14 Song X M. Sampling Techniques (in Chinese). Beijing: China Forestry Publishing House, 1995

15 Thompson S K, Seber G A F. Adaptive Sampling. New York: Wiley, 1996

16 Christman M C, Lan F. Inverse adaptive cluster sampling. Biometrics, 2001, 57: 1096-1105

17 Rocco E. Constrained inverse adaptive cluster sampling. J Off Stat, 2003, 19: 45-57

18 Brown J A, Manly B J F. Restricted adaptive cluster sampling. Environ Ecol Stat, 1998, 5: 49-63

Open Access This article is distributed under the terms of the Creative Commons Attribution License which permits any use, distribution, and reproduction in any medium, provided the original author(s) and source are credited. 